\title{
Apendicitis aguda perforada secundaria a una tuberculosis como causa de sepsis posparto
}

\section{Acute perforated appendicitis secondary to tuberculosis as a cause of postpartum infection}

\author{
Natalia Rodríguez ${ }^{1}$ y Natalia Gómez ${ }^{2}$ \\ 'Universidad Pontificia Bolivariana, Medellín, Colombia. \\ ${ }^{2}$ Clínica Universitaria Bolivariana, Medellín, Colombia. \\ Sin financiamiento. \\ Conflicto de interés: los autores no tienen ningún conflicto de interés.
}

Recibido: 2 de julio de 2019 / Aceptado: 24 de febrero de 2020

\section{Resumen}

La tuberculosis gastrointestinal es una manifestación extrapulmonar poco frecuente, correspondiendo al $3 \%$ de los casos y que compromete principalmente la región ileocecal; la afectación apendicular es poco frecuente. La apendicitis tuberculosa se puede presentar como un cuadro agudo indistinguible de las otras causas de apendicitis y el diagnóstico generalmente se hace por el hallazgo histopatológico, lo que retrasa el tratamiento y puede llevar a complicaciones. El período posparto es de riesgo de una reactivación de una tuberculosis, debido a cambios en el sistema inmunológico que se manifiestan como un síndrome de reconstitución inmune. Se presenta el caso de una paciente puérpera que ingresa por un choque séptico secundario a una apendicitis perforada. La histopatología demostró una inflamación granulomatosa crónica, con posterior confirmación por biología molecular de una tuberculosis pulmonar.

Palabras clave: apendicitis; abdominal; intestinal; tuberculosis; posparto.

\section{Introducción}

L a tuberculosis $(\mathrm{TBC})$ es una de las diez principales causas de muerte en el mundo y la principal causa por un único agente infeccioso. En el año 2017, se estimaron 1,3 millones de muertes por TBC en pacientes sin infección por VIH y 300.000 muertes adicionales en pacientes con VIH, con un estimado de 10 millones de casos nuevos. Según la OMS, en Colombia en el año 2017 hubo una incidencia de 16.000 casos de TBC lo que equivale a 33 casos/100.000

\begin{abstract}
Gastrointestinal tuberculosis is a rare extrapulmonary manifestation, it represents $3 \%$ of cases and mainly involves the ileocecal region; appendiceal involvement is rare. Tuberculous appendicitis can present as an acute condition indistinguishable from other causes of appendicitis and the diagnosis is generally made by histopathological finding, which delays treatment and can lead to complications. The postpartum period is at risk of a reactivation of tuberculosis, due to changes in the immune system that manifests as an immune reconstitution syndrome. We present the case of a postpartum patient admitted for septic shock secondary to perforated appendicitis, the pathology reported chronic granulomatous inflammation and subsequent confirmation by molecular technique of pulmonary tuberculosis.

Keywords: appendicitis; abdominal; intestinal; tuberculosis; postpartum.
\end{abstract}

habitantes, con 1.300 muertes en pacientes sin VIH y 430 muertes adicionales en pacientes con $\mathrm{VIH}^{1}$.

Aunque la TBC puede afectar cualquier órgano, el sitio más comúnmente comprometido es el pulmón ${ }^{2}$. El sistema gastrointestinal es uno de los menos prevalentes, correspondiendo al 3\% de las TBC extrapulmonares. La región que más frecuentemente se ve afectada es la ileocecal, mientras que la TBC apendicular es rara, siendo reportada en el 0,1 a 3\% de los pacientes sometidos a una apendicectomía ${ }^{3}$. Los síntomas y hallazgos al examen físico son inespecíficos e indistingui- 
bles de las otras causas de apendicitis lo cual retrasa el diagnóstico hasta la confirmación histopatológica.

El embarazo se caracteriza por un estado de inmunosupresión que es vital para el mantenimiento de la gestación y así evitar el rechazo del feto ${ }^{4}$. Normalmente, se presenta un aumento en la respuesta $\mathrm{TH} 2$ con disminución en la respuesta $\mathrm{TH} 1$ y en la inmunidad mediada por células. Posterior al parto, la supresión TH1 se revierte y se produce una reactivación linfocitaria que lleva al inicio de síntomas, lo que sugiere una especie de síndrome de reconstitución inmune 5 .

Debido a lo infrecuente del cuadro y el reto diagnóstico que implica, se presenta el caso de una paciente puérpera con un choque séptico secundario a una apendicitis aguda perforada de etiología tuberculosa.

\section{Caso clínico}

Mujer de 21 años sin antecedentes patológicos, con antecedentes de un parto de vértice espontáneo un mes previo a su ingreso. Consultó por un cuadro clínico de 10 días de evolución consistente en dolor abdominal localizado en el epigastrio con posterior irradiación al hemiabdomen inferior, constante, de moderada intensidad, asociado a diarrea entre cuatro a cinco episodios al día, sensación febril y malestar general. Negaba síntomas respiratorios o urinarios. Ingresó con hemodinamia inestable, con una presión arterial 75/45 $\mathrm{mm} \mathrm{Hg}$, frecuencia cardíaca: 118 por minuto, $\mathrm{T}^{\mathrm{o}}$ axilar: $34,5^{\circ} \mathrm{C}$, con palidez generalizada y signos de hipoperfusión. En el examen físico presentaba distensión abdominal, con dolor a la palpación generalizada principalmente en región de fosa ilíaca derecha, defensa muscular y signos de irritación peritoneal. Se inició reanimación con reposición de volumen y antibioticoterapia con piperacilina/tazobactam intravenoso 4,5 gramos cada ocho horas. Entre los exámenes de laboratorio iniciales destacó un hemograma con una hemoglobina de $8,2 \mathrm{mg} / \mathrm{dL}$, sin leucocitosis, con linfopenia y $\sin$ trombocitopenia, una PCR $31 \mathrm{mg} / \mathrm{dL}$, y prolongación de los tiempos de coagulación.

Fue evaluada por cirugía, sospechándose una apendicitis perforada. Se realizó una laparotomía exploratoria cuyos hallazgos intraoperatorios fueron un gran plastrón apendicular en el hipogastrio con compromiso de epiplón y fondo del útero, hemorragia en capas en la región posterior del útero, signos de peritonitis con líquido seropurulento y una colección pélvica purulenta. Se realizó la liberación de adherencias, drenaje en los cuatro cuadrantes y de la colección pélvica y apendicectomía. Por sangrado uterino se realizó empaquetamiento y se dejó una laparotomía contenida. Fue trasladada grave a la unidad de cuidados intensivos, con apoyo ventilatorio y vasopresor con norepinefrina.

Los cultivos de líquido peritoneal demostraron creci- miento de Escherichia coli resistente a ampicilina/sulbactam y Enterococcus casseliflavus sensible a ampicilina. A las 24 horas fue llevada a un desempaquetamiento, aseo quirúrgico y cierre de la pared abdominal. Los cultivos de la segunda intervención fueron negativos.

La evolución en los primeros días fue favorable, con disminución progresivo del soporte vasopresor y ventilatorio, hemodinamia estable, afebril, con reducción de los reactantes de fase aguda. Sin embargo, después de la primera semana la paciente comenzó a presentar fiebre. Se realizó una tomografía (TC) de abdomen contrastada que evidenció una hepatomegalia, una colección pélvica derecha y otra pequeña colección multiloculada en el flanco izquierdo. Las imágenes que alcanzaban a visualizarse del tórax mostraron unas consolidaciones segmentarias en ambos lóbulos inferiores, compatibles con micronódulos centrolobulillares confluentes, sugerentes de una infección de vía aérea pequeña del tipo TBC (Figuras 1 y 2).

Inicialmente se tuvo una baja sospecha de infección por TBC, pues la paciente negaba síntomas respiratorios o constitucionales y no tenía antecedentes epidemiológicos de contactos; por lo que los hallazgos pulmonares fueron interpretados como secundarios a microaspiraciones y que la fiebre estaba asociada a los antibacterianos. Una vez completado el tratamiento, se suspendió piperacilina/ tazobactam.

La evolución de la paciente no fue satisfactoria, con

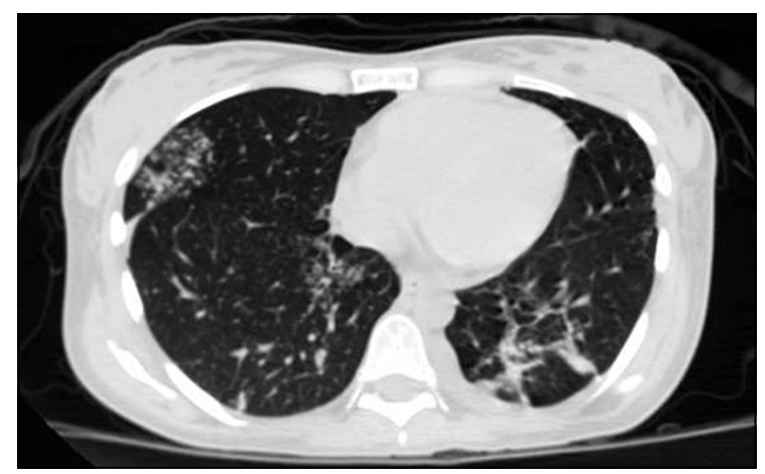

Figura 1. Tomografía de abdomen contrastada con extensión a tórax. Micronódulos centrolobulillares confluentes en lóbulos inferiores.

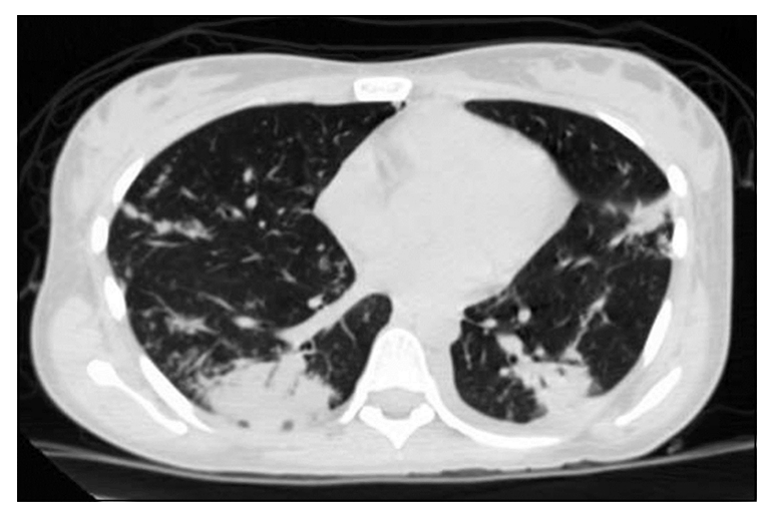

Figura 2. Tomografía de abdomen contrastada con extensión a tórax. Consolidaciones segmentarias en ambos lóbulos inferiores. 
fiebre intermitente y una nueva elevación de los reactantes de fase aguda. El informe anatomopatológico definitivo del apéndice demostró una apendicitis aguda purulenta abscedada con peritonitis asociada, y presencia de granulomas epitelioides con necrosis central rodeados por células mononucleares y células gigantes multinucleadas tipo Langhans.

Se reevaluó el tórax con una TC que mostró persistencia de los cambios en el parénquima pulmonar, por lo que se realizó una fibrobroncoscopia con lavado broncoalveolar, obteniendo un GeneXpert MTB/RIF positivo para la detección de ADN de Mycobacterium tuberculosis $\sin$ resistencia a rifampicina.

Se diagnosticó una TBC diseminada con compromiso pulmonar y apendicular; sin signos o síntomas de otro sistema. La paciente tenía una prueba serológica de VIH negativa en sus controles prenatales. Después de varias sesiones de interrogaciones, la paciente reveló que recientemente su marido había vuelto a la casa después de pasar un período en la cárcel donde habría sido diagnosticado con una TBC.

La paciente inició tratamiento con isoniazida, rifampicina, pirazinamida y etambutol más piridoxina, con adecuada tolerancia.

La evolución fue satisfactoria, sin fiebre y otros síntomas, por lo que fue dada de alta, sin complicaciones conocidas hasta el momento de esta publicación. El recién nacido fue internado descartándose una TBC, por lo que también fue dado de alta.

\section{Discusión}

La TBC abdominal incluye el compromiso del tracto gastrointestinal, peritoneal y vísceras asociadas; es la sexta forma más frecuente de TBC extrapulmonar. Se puede manifestar en cualquier localización del tracto gastrointestinal, siendo la región ileocecal la más afectada; entre 44 y $93 \%$ de los $\operatorname{casos}^{6}$.

La apendicitis tuberculosa es reportada en $0,1-3 \%$ de los pacientes sometidos a apendicectomía ${ }^{3}$. En 1987, Gupta y cols. revisaron los análisis histopatológicos de 2.921 apendicectomías realizadas en un lapso de 25 años en India, 70 muestras (2,3\%) tenían hallazgos típicos de $\mathrm{TBC}^{7}$. En 2004, Agarwal y cols. evaluaron retrospectivamente las apendicectomías realizadas entre 1991-2000 en otro hospital de India $(n=870)$, encontrando 26 casos $(2,9 \%)$ de apendicitis tuberculosa ${ }^{8}$. En 2011, Akbulut y cols. revisaron las apendicectomías realizadas entre 2006-2010 en un hospital de Turquía. De un total de 5.262 muestras, 56 (1\%) tenía histología anormal y dos muestras fueron compatibles con TBC $(0,03 \%$ de los casos totales) $)^{9}$.

En una revisión de la literatura científica, de un com- pilado de 80.998 muestras de apendicectomías realizadas entre 2000-2010, 1.366 (2,5\%) presentaron hallazgos anormales, y de estos 35 casos $(0,04 \%$ de total) correspondieron a $\mathrm{TBC}^{10}$. Finalmente, en 2018 Jolayemi y cols. realizaron un estudio retrospectivo de las apendicectomías realizadas entre 2012-2015 en un hospital de Sudáfrica $(\mathrm{n}=290)$, encontrando dos casos de TBC apendicular $(0,7 \%)^{10}$.

Con respecto a la epidemiología de la TBC en el puerperio, en 2013 Cheng y cols. realizaron una búsqueda sistemática de reportes de TBC periparto encontrando 29 casos; el promedio de inicio de los síntomas fue de cuatro días posterior al parto, con $75 \%$ de las pacientes con síntomas en los primeros 10 días. La mayoría tenían compromiso extrapulmonar, siendo el más frecuente la meningitis tuberculosa; solo se encontró un caso de compromiso abdominal sin especificar la localización ${ }^{4}$.

En 2012, Zenner y cols. revisaron la epidemiología de la TBC en el parto y puerperio entre 1996 y 2008; se incluyeron 192.801 mujeres de las cuales 177 presentaron TBC. Se encontró que la mayoría de los casos ocurrieron en los siguientes 180 días posteriores al parto, con una incidencia de 19,2 casos/100.000 personas-año y una razón de tasa de incidencias de 1,95; la manifestación extrapulmonar fue la más frecuente, siendo el tracto genitourinario y el sistema linfático los sitios más comúnmente afectados ${ }^{11}$. No se encontraron casos de compromiso apendicular por TBC en el período posparto.

En Colombia, Ávila y cols. realizaron un análisis de los apéndices extraídos en Boyacá. De un total de 1.688 muestras se encontraron dos casos de TBC $(0,1 \%)^{12}$. No se encontraron en nuestro medio más reportes de compromiso apendicular por TBC, y ninguno de los dos casos fue en pacientes posparto.

$\mathrm{La}$ apendicitis tuberculosa puede ocurrir como un proceso primario o secundario. En la forma secundaria hay evidencia de infección por TBC en otros sistemas y el apéndice se infecta a través de diseminación hematógena, diseminación linfática, por contigüidad o ingesta de esputo infectado. La forma primaria o aislada, que ocurre aún con menor frecuencia, se establece cuando no hay evidencia de infección en otros órganos y se produciría por la ingesta de comida contaminada ${ }^{2,3}$. En el caso de nuestra paciente hubo evidencia de compromiso pulmonar, constituyendo un proceso secundario.

Se han descrito tres formas de presentación clínica de la apendicitis tuberculosa: la crónica, que se caracteriza por episodios intermitentes de dolor abdominal en la fosa ilíaca derecha, diarrea, fiebre y pérdida de peso; aguda, la cual produce un cuadro clínico indistinguible de las otras causas de apendicitis; y la latente, cuando es evidenciada como un hallazgo incidental en una apendicectomía electiva ${ }^{13,14}$. La presentación aguda puede verse precipitada por una sobreinfección piógena 5 . En el caso de nuestra 
paciente, el cuadro fue el de una apendicitis aguda, con perforación y sobreinfección bacteriana.

En la mayoría de los casos no hay síntomas o signos clásicos que orienten hacia una apendicitis tuberculosa, $\mathrm{y}$ es el informe histopatológico el que conduce al diagnóstico ${ }^{2}$. Los hallazgos histopatológicos clásicos son la presencia de necrosis de caseificación y células gigantes de Langhans ${ }^{15}$. El diagnóstico microbiológico es difícil debido a la baja sensibilidad que tienen las baciloscopias y el cultivo. El desarrollo de técnicas moleculares de amplificación del material genético ha aumentado la confirmación diagnóstica ${ }^{2}$.

Ocasionalmente, los estudios imagenológicos pueden orientar hacia el diagnóstico cuando se evidencia una masa inflamatoria en la zona ileocecal, con engrosamiento asimétrico de la válvula, ganglios linfáticos con realce regional, ascitis y engrosamiento mesentérico ${ }^{14}$. Siempre debe evaluarse si existe compromiso pulmonar, ya que esto orienta sobre el riesgo de transmisión y sirve como guía para el diagnóstico y tratamiento ${ }^{16}$.

El manejo se basa en el tratamiento farmacológico antituberculoso usando el mismo esquema que en la TBC pulmonar (dos meses de isoniazida/rifampicina/pirazinamida/etambutol, seguidos de cuatro meses de isoniazida/ rifampicina), en conjunto con el manejo quirúrgico ${ }^{2}$.

Durante el embarazo se produce un estado inmunosupresor que es crítico para el mantenimiento de la gestación. Este estado se caracteriza por un aumento en las respuestas TH2 y TH3, con una disminución en las citoquinas TH1 que son deletéreas para el feto; se ha evidenciado además una disminución en la reactividad linfocitaria a los antígenos de proteína purificados de tuberculina, lo que indica una supresión de la inmunidad mediada por células. Esto puede llevar a que se produzca una reactivación de un foco endógeno de TBC que permanece subclínico o quiescente hasta que finaliza el embarazo. En el posparto, la reactividad linfocitaria regresa a la normalidad en las primeras 24 horas y se recupera completamente a las cuatro semanas. Esto favorece la aparición de nuevos casos de TBC o la reactivación de casos preexistentes, lo que sugiere que la exacerbación de la TBC durante el posparto estaría relacionada con reconstitución de la respuesta inmune a antígenos específicos del patógeno ${ }^{4,5}$.

En conclusión, la TBC es una causa infrecuente de apendicitis de presentación inespecífica, lo que lleva a un retraso en el diagnóstico, tratamiento y mayor riesgo de complicaciones. El diagnóstico generalmente se realiza por el hallazgo histopatológico. Sin embargo, a pesar de la baja frecuencia, sigue siendo una causa de apendicitis que debe tenerse en cuenta dentro de los posibles diagnósticos diferenciales, principalmente en zonas endémicas, en pacientes con cuadros crónicos y síntomas pulmonares asociados, o con evoluciones clínicas no satisfactorias. Adicionalmente, las mujeres puérperas tienen un mayor riesgo de presentar una reactivación de una TBC por los cambios inmunológicos del embarazo, con alta incidencia de manifestaciones extrapulmonares y con diagnóstico y tratamiento efectivo tardíos, lo que aumenta la morbimortalidad.

\section{Referencias bibliográficas}

1.- Global tuberculosis report 2018. Geneva: World Health Organization; 2018. Licence: CC BYNC-SA 3.0 IGO. Disponible en: https://apps. who.int/medicinedocs/documents/s23553en/ s23553en.pdf

2.- Barbagallo F, Latteri S, Sofia M, Ricotta A, Castello G, Chisari A, et al. Appendicular tuberculosis: the resurgence of an old disease with difficult diagnosis. World J Gastroenterol 2010; 16: 518-21.

3.- Moniri A, Marjani M, Tabarsi P, Baghaei P, Dorudinia A, Masjedi M R. Multidrug-resistant tubercular appendicitis: Report of a case. Int $\mathrm{J}$ Mycobacteriol 2013; 2: 227-9. doi: 10.1016/j. ijmyco.2013.07.003.

4.- Cheng V C, Woo P C, Lau S K, Cheung C H, Yung R W, Yam L Y, et al. Peripartum tuberculosis as a form of immunorestitution disease. Eur J Clin Microbiol Infect Dis 2003; 22: 313-7.

5.- Singh N, Perfect J R. Immune reconstitution syndrome and exacerbation of infections after pregnancy. Clin Infect Dis 2007; 45: 1192-9.
6.- Choi E H, Coyle W J. Gastrointestinal tuberculosis. Microbiol Spectr 2016; 4(6): doi: 10.1128/microbiolspec.TNMI7-0014-2016.

7.- Gupta S C, Gupta A K, Keswani N K, Singh P A, Tripathi A K, Krishna V. Pathology of tropical appendicitis. J Clin Pathol 1989; 42: 1169-72.

8.- Agarwal P, Sharma D, Agarwal A, Agarwal V, Tandon A, Baghel K D, et al. Tuberculous appendicitis in India. Trop Doct 2004; 34: 36-8.

9.- Akbulut S, Tas M, Sogutcu N, Arikanoglu Z, Basbug M, Ulku A, et al. Unusual histopathological findings in appendectomy specimens: a retrospective analysis and literature review. World J Gastroenterol 2011; 17: 1961-70. doi: 10.3748/wjg.v17.i15.1961.

10.- Jolayemi O O, Moodley N B, Kong V Y, Tlou B, Bruce J L, Clarke D L. The usefulness of routine histological examination of appendicectomy specimens in a South African tertiary centre. S Afr Med J 2018; 108: 342-6. doi: 10.7196/SAMJ.2017. v108i4.12759.

11.- Zenner D, Kruijshaar M E, Andrews N,
Abubakar I. Risk of tuberculosis in pregnancy: a national, primary care-based cohort and selfcontrolled case series study. Am J Respir Crit Care Med 2012; 185: 779-84. doi: 10.1164/ rccm.201106-1083OC.

12.- Ávila M J, García-Acero M. Apendicitis aguda: revisión de la presentación histopatológica en Boyacá, Colombia. Rev Colomb Cir 2015; 30 : 125-30.

13.- Chong V H, Telisinghe P U, Yapp S K, Chong C F. Tuberculous appendix: a review of clinical presentations and outcomes. Singapore Med J 2011; 52: 90-3.

14.- Akbulut S, Yagmur Y, Bakir S, Sogutcu N, Yilmaz D, Senol A, et al. Appendicular tuberculosis: review of 155 published cases and a report of two cases. Eur J Trauma Emerg Surg 2010; 36: 579-85. doi: 10.1007/s00068-0100040-y.

15.- Na A F, Brown S, Chandra R. Appendiceal tuberculosis. ANZ J Surg 2017; 87: E102-E103. doi: 10.1111/ans.13016.

16.- Constantinescu C, Vayalumkal J, Fisher D. An unusual case of appendicitis. CMAJ 2014; 186: 1241-3. doi: 10.1503/cmaj.131126. 